\title{
Microstrain and residual stress in thin-films made from silver nanoparticles deposited by inkjet-printing technology
}

\author{
Romain Cauchois ${ }^{1, a *}$, András Borbély ${ }^{2, b}$, Patrice Gergaud ${ }^{3, c}$, \\ Mohamed Saadaoui ${ }^{4, d}$ and Karim Inal ${ }^{5, e}$ \\ ${ }^{1}$ Holst Centre, TNO, High Tech Campus 31, 5605 KN Eindhoven - The Netherlands \\ ${ }^{2}$ Ecole Nationale Supérieure des Mines, SMS, 158 cours Fauriel, 42023 St-Etienne - France \\ ${ }^{3}$ CEA LETI, MINATEC campus, 17 rue des Martyrs, 38054 Grenoble Cedex 9 - France \\ ${ }^{4}$ Ecole Nationale Supérieure des Mines, CMP, 880 avenue de Mimet, 13541 Gardanne - France \\ ${ }^{5}$ Mines ParisTech, CEMEF-UMR 7635, 1 rue Claude Daunesse, 06904 Sophia Antipolis - France \\ aromain.cauchois@tno.nl, bandreas.borbely@mines-stetienne.fr, ${ }^{\mathrm{b}}$ patrice.gergaud@cea.fr, \\ dmohammed.saadaoui@mines-stetienne.fr , ${ }^{\mathrm{e}}$ karim.inal@mines-paristech.fr
}

Keywords: in-situ XRD, Ag nanoparticle, polycrystalline thin films, inkjet printing

\begin{abstract}
Colloidal suspensions of nanoparticles are increasingly employed in the fabrication process of electronic devices using inkjet-printing technology and a consecutive thermal treatment. The evolution of internal stresses during the conversion of silver nanoparticle-based ink into a metallic thin-film by a thermal sintering process has been investigated by in-situ XRD using the $\sin ^{2} \psi$ method. Despite the CTE mismatch at the film/substrate interface, the residual stress in silver films (below $70 \mathrm{MPa}$ ) remains lower than in conventional PVD thin-films, as a result of the remaining porosity. A Warren-Averbach analysis further showed that the crystallite growth is associated with a minimization of the twin fault density and the elastic microstrain energy above $150^{\circ} \mathrm{C}$. A stabilization of the microstructure and internal stress is observed above $300^{\circ} \mathrm{C}$. Inkjetprinting technology thus appears as a good alternative to conventional metallization techniques and offers significant opportunities asset for interconnect and electronic packaging.
\end{abstract}

\section{Introduction}

Analysis of residual stress in thin films is of utmost importance because of its crucial influence on product reliability, as well as on induced local properties. In particular, residual stress can be introduced during the film processing, due to the different thermo-mechanical properties of materials, to the variation of microstructure, or to geometry. Several non-destructive methods have been suggested to characterize the residual stress in thin films, which ranges from common techniques such as curvature measurement (Stoney) [1] or X-ray diffraction (XRD) [2], to more specific approaches such as Raman scattering [3]. Given the increasing degree of miniaturization reached in current micro- and nanotechnologies, it is of major importance to deploy these experimental techniques allowing multi-scale stress analysis.

It is ever more important that new alternatives to lithographic process are under development to make thin films on large-areas or mesoscale patterns with fine pitch [4]. In particular, direct printing of metallic colloidal suspensions has received steadily growing interest for both scientific and industrial applications [5-7]. In the past few years, the technologies using metallic nanoparticles underwent major progresses thanks to achievements in inks development, printing and sintering tools. Nanoparticles are known for their specific optical, mechanical, and thermodynamic properties derived from high surface-to-volume ratio [8-10]. Properties of thin films made from sintered nanoparticles are related to structural parameters as grain size, grain boundary types and crystalline defects, whose evolution strongly depends on the applied heat treatment, i.e. temperature, time and heat ramp. Nonetheless, understanding the physics of nanoclusters sintering is still a challenging task. 
X-ray diffraction proved to be widely used for determination of residual stress in thin films coatings by probing directly crystallographic structures. In this paper, the evolution of internal stress during the conversion of silver nanoparticle-based ink into a metallic thin film has been investigated by insitu XRD using the $\sin ^{2} \psi$ method $[11,12]$, which relates the evolution of interplanar spacing to XRD peaks shift and to the underlying stress. The microstructure of such thin films has been further studied by analyzing the broadening of high-resolution X-ray peaks and correlating peak breadth to average crystallite size and lattice microstrain using a Warren-Averbach analysis [13].

\section{Experimental}

In this study, a commercially available colloidal suspension with a silver nanoparticle content of $20 \mathrm{wt} \%$ was purchased from Sun Chemical. Nanoparticles have an average diameter of 25-30 nm. They are dispersed in a liquid vehicle composed of polar solvents: ethanol, ethylene glycol and glycerol. Additionally, in order to avoid agglomeration and sedimentation, silver nanoparticles are coated with a capping agent. A thin film of this colloidal dispersion is deposited onto a n-type (100) silicon substrate using a drop-on-demand inkjet printing technology. Deposition was performed at room temperature using a 256 nozzle-printhead integrated in an in-line prototype developed in the lab (JetPac) [14]. A consecutive thermal treatment is finally applied in order to evaporate solvents and to initiate the sintering process.

Electrical conduction in printed thin-films is achieved by a sintering step through Rapid Thermal Annealing (RTA). Curing was conducted in a $\mathrm{N}_{2}$ atmosphere with halogen lamps in a JetFirst 100 oven from Jipelec. All else being equal, inkjet-printed thin films were cured in a RTA with varying thermal ramps - from 0.1 to $150^{\circ} \mathrm{C} / \mathrm{s}$ - and sintering times - from 15 to $60 \mathrm{~min}$. For the $15 \mathrm{~min}$ treatments, effect of temperature was investigated at $150{ }^{\circ} \mathrm{C}, 300^{\circ} \mathrm{C}$ and $500^{\circ} \mathrm{C}$ with a thermal ramp of $10^{\circ} \mathrm{C} / \mathrm{s}$. Typical thickness of thin films after thermal processing was about $330 \mathrm{~nm}$.

In-situ XRD stress analysis using the $\sin ^{2} \psi$ method have been performed on a four circles Xpert MRD PANalytical diffractometer equipped with a sealed copper tube. The primary optic is a polycapillary X-ray lens and the secondary optic is a parallel plate collimator with an angular aperture of $0.18^{\circ}$. The heating system is a DHS1100-Anton Paar furnace with a graphite dome. The temperature is increased and then cooled down by step of $20^{\circ} \mathrm{C}$ with a heating/cooling ramp of $200^{\circ} \mathrm{C}$ per second between two thermal steps. The strain measurements are done following the shifts of the $\mathrm{Ag}(111)$ reflections at seven tilt angles between 0 and $71^{\circ}$. For ex-situ measurements the (113) reflections are used. The stress analysis is performed assuming an isotropic biaxial state of stress in the plane of the film. The elastic constants of bulk silver are used.

Stress and X-ray peak profile measurements were performed with PANalytical MRD and MPD PRO diffractometers, respectively. To reduce instrumental effects, the latter was foreseen with a parabolic X-ray mirror coupled to a double-bounce channel-cut monochromator (Ge, 220) preset for $\mathrm{Cu}-\mathrm{K} \alpha 1$ radiation. The diffracted beam was measured with linear PIXcel detector. The particle size, the planar fault density and the microstrain were determined according to the Warren-Averbach analysis using the 111/222 and 200/400 reflection-pairs.

\section{Results and discussions}

Since residual stress is highly correlated to process conditions, and the microstructure, X-ray measurements were performed to assess the impact of process parameters on residual stress. The results show that the contribution from thermal ramp and sintering time is almost negligible since the stress variation is comprised within a range of $10 \mathrm{MPa}$ (see Table 1). The low stress (20-30 $\mathrm{MPa}$ ) observable in such printed structures after annealing has to be emphasized because of its great difference with the expected behavior of conventional thin films during thermal cycles. If the low impact of sintering time complies with a stabilization of microstructure observed after 15 min of curing, the reason of the limited impact of thermal ramp remains unclear. Indeed, as seen in previous reports, both grain and pore sizes are affected by the thermal ramp but these growths seem insufficient to affect the residual mechanical state. 
Table 1: Values of residual stress, after a $300^{\circ} \mathrm{C}$ sintering cycle, showing the low impact of both sintering ramp and sintering time.

\begin{tabular}{lcccc||cc}
\hline Sintering ramp $\left[{ }^{\circ} \mathrm{C} / \mathrm{s}\right]$ & 0.1 & 10 & 50 & 150 & 10 & 10 \\
Sintering time $[\mathrm{min}]$ & 15 & 15 & 15 & 15 & 45 & 60 \\
\hline Residual stress $[\mathrm{MPa}]$ & $23 \pm 3$ & $22 \pm 0.6$ & $33 \pm 1.5$ & $23 \pm 2$ & $21 \pm 2.5$ & $29 \pm 1$ \\
\hline
\end{tabular}

In order to explain these values, in-situ diffraction during the heating and cooling steps was performed. Hodge et al. [15] have followed the evolution of internal stress with temperature for both sputtered and electroplated silver thin films of $150 \mathrm{~nm}$ thickness deposited on a silicon substrate. As shown on Fig. 1a, the residual stress in thermally processed films after the initial heating is higher than in freshly deposited films in both cases. While initial stress is tensile, an elastic deformation of the film is occurring during the curing until reaching the elastic limit when irreversible deformation arises. As expected from the Hall-Petch equation [16], the yield stress is highly dependent on the microstructure and especially on the average grain size of the deposit. Since the average grain size is smaller in sputtered films than in electroplated films, the thermoelastic regime is extended up to $200^{\circ} \mathrm{C}$ (vs. $100^{\circ} \mathrm{C}$ for electroplated films, according to Fig. 1a). Electroplated films are indeed highly reactive and subject to substantial grain growth during thermal curing. Above the yield stress, the coefficient of thermal expansion (CTE) mismatch is accommodated by the activation of several relaxation mechanisms such as vacancy consumption, dislocation annihilation, recovery, recrystallization, grain growth via boundary sliding, or creep.

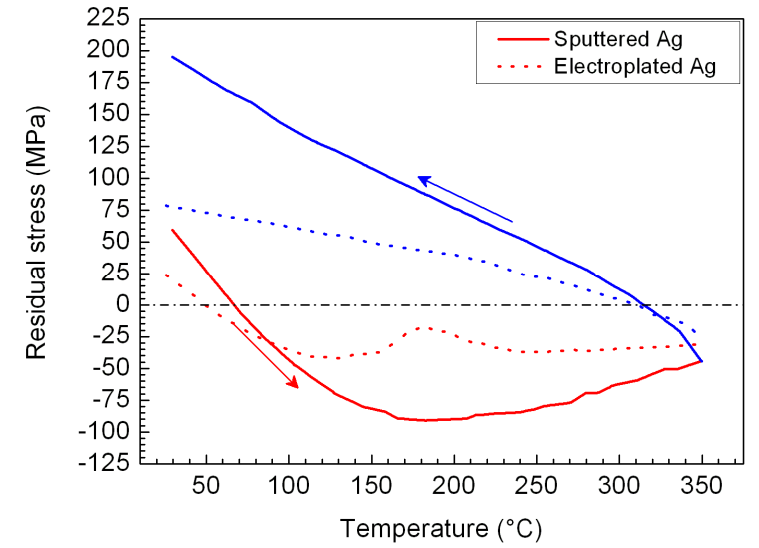

(a)

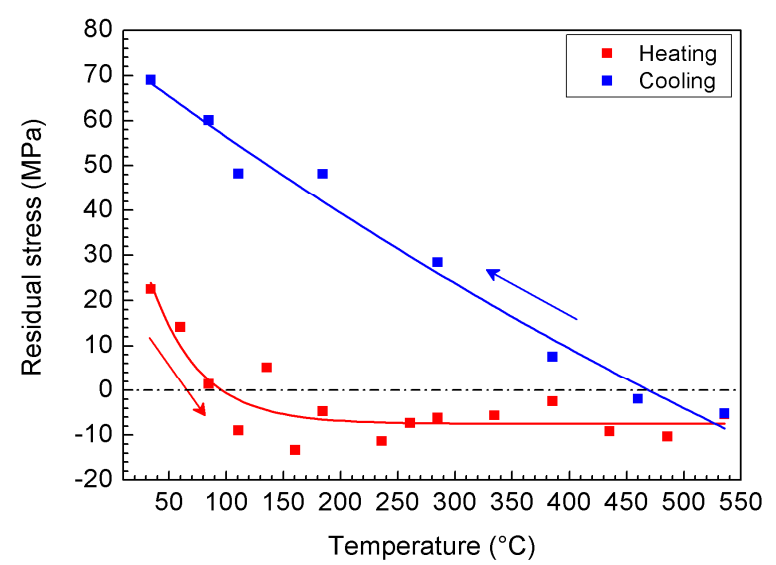

(b)

Figure 1: Internal stress in $200 \mathrm{~nm}$-thick sputtered and electroplated silver thin films using the Stoney method (a) adapted from [15], and in our $330 \mathrm{~nm}$-thick inkjet-printed thin film using in-situ XRD measurements (b) [present work]. All films are deposited on a silicon substrate.

Internal stress evolution in inkjet-printed films on silicon substrate was investigated using in-situ Xray diffraction and is presented in Fig. $1 \mathrm{~b}$ for a thermal cycle up to $550^{\circ} \mathrm{C}$. Due to experimental limitations, a gentle drying at $60^{\circ} \mathrm{C}$ has been carried out in order to remove a significant part of the solvent phase and to avoid ink flowing off from the substrate when placed vertically. The first conclusion that can be emphasized is the low residual stress level within the printed films, similar to the stress level observed in the electroplated silver films. The residual stress of $70 \mathrm{MPa}$ in the nanoparticulate film is three times lower than in a $200 \mathrm{~nm}$-thick polycrystalline silver film deposited by sputtering. The similarity with the unstable and highly scalable structure of electroplated silver films gives credit to results. However, the internal stress generated within the inkjet-printed silver thin film is quite unlike the one developed in electroplated films. The linear branch observed during the cool down of our films indeed tends to indicates that the nanoparticles do not flow while conventional films are dominated by plastic deformation and flow stresses during cool down [17]. In electroplated films, the stress of $75 \mathrm{MPa}$ observable after a curing at $350^{\circ} \mathrm{C}$ would result by 
extrapolation in a stress of about $150 \mathrm{MPa}$ after a curing at $550^{\circ} \mathrm{C}$, which is twice as much as the measured value in printed films. As shown in Fig. 1b, the inkjet-printed silver film is initially in tension, which has to be related to the drying step of the sample before the experiment to materialize a viscous/solid film, but without initiating any sintering mechanism. As demonstrated by Hoffman et al., when adjacent grains are close enough, grain boundaries are formed between impinging islands through the reduction of surface energy [18]. Thus, the formation of a continuous film by gap closing, during this preliminary curing, generates a tensile stress with the help of the relative mismatch in thermal expansion between the film and the substrate.

During the heating step, residual solvents progressively evaporate and adhesion between nanoparticles and the substrate is initiated. As a consequence, stress decreases and becomes compressive at about $100^{\circ} \mathrm{C}$, just as in sputtered or electroplated silver films. But unlike PVD films, the initial slope of the stress cycle is uncorrelated to a thermoelastic behavior since the film is not yet fully formed and adherent to the substrate. The initial slope of the curve is much smaller than that of the film's thermoelastic behavior, given by the associated stress:

$$
\frac{d \sigma}{d T}=\left(\alpha_{s}-\alpha_{f}\right) \frac{E_{f}}{1-v_{f}} \text {. }
$$

where $\alpha_{s}$ and $\alpha_{f}$ are respectively the coefficients of thermal expansion of the silicon substrate $\left(\alpha_{s}=\right.$ $\left.3 \cdot 10^{-6} \mathrm{~K}^{-1}\right)$ and of the film $\left(\alpha_{f}=19 \cdot 10^{-6} \mathrm{~K}^{-1}\right)$, and $E / 1-v$ is the biaxial modulus of the porous silver composite. With the sole contribution of CTE mismatch between the substrate and the film, the stress of a fully dense silver film would be $-115 \mathrm{MPa}$ at $100^{\circ} \mathrm{C}$, assuming an initial stress-free state. During heating, above $100^{\circ} \mathrm{C}$, the nanoparticulate silver film can be considered as stress free. As seen previously, it is assumed that, between $100^{\circ} \mathrm{C}$ and $550^{\circ} \mathrm{C}$, the tensile stress induced by thermal expansion mismatch is mitigated not only by recrystallization/recovery but also by atomic mass transport associated with the sintering phenomenon. The presence of porosities plays also a key role in the accommodation of the stress by decreasing the average CTE and elastic properties of this porous composite system. The interpretation is in good agreement with the microstructure stabilization above $300^{\circ} \mathrm{C}$ as deduced from the peak width evolution (Fig. 2a). Using the Scherrer equation as a preliminary evaluation, the average crystallite size deduced from the peak width evolves from $25 \mathrm{~nm}$ for the as-deposited layer, to $120 \mathrm{~nm}$ after thermal curing.

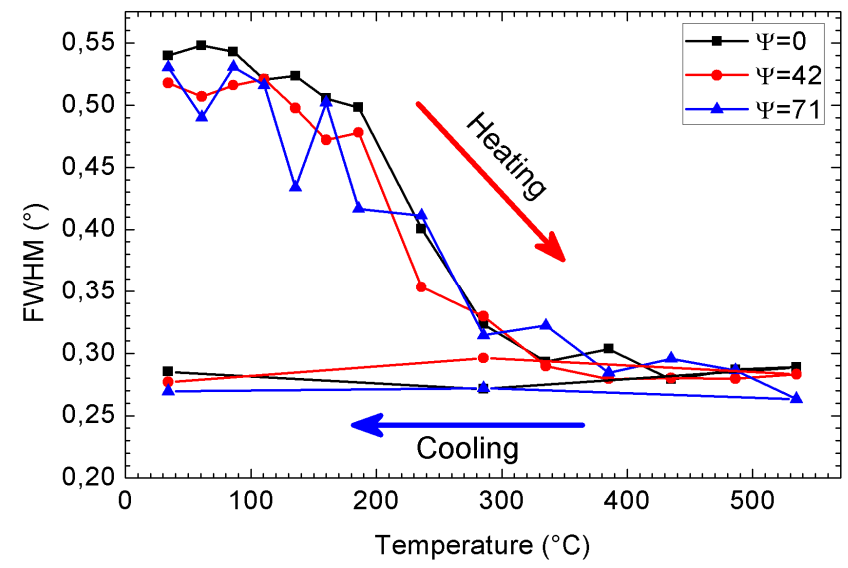

(a)

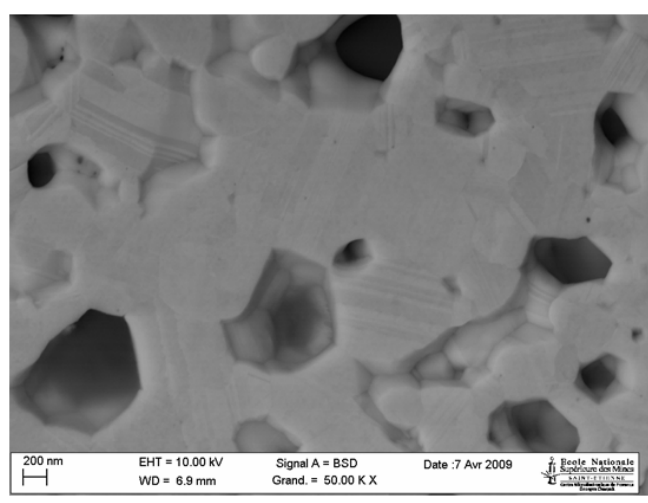

(b)

Figure 2: Full Width at Half Maximum (FWHM) of the 111 peak of $\mathrm{Ag}$ as a function of temperature at several $\psi$ angles (a), and SEM image of the printed silver film at the interface with the silicon substrate, after peeling off the film (b).

The final residual stress is mainly generated during cooling. In our case, the microstructure is stabilized from $300^{\circ} \mathrm{C}$ but the interface is not perfect. Porosity is clearly observable (with a characteristic length up to $1 \mu \mathrm{m}$ ) and grains have been flattened at the interface with the silicon substrate (see Fig. 2b). As a consequence, the stress generated at the interface due to thermal 
mismatch is quickly accommodated by the layer without transferring the load due to CTE. Strain can also be locally further accommodated due to the free surfaces related to porosity.

Bolstered by these in-situ measurements performed on a specific sintering condition at $550^{\circ} \mathrm{C}$, the behavior of the internal stress during the thermal cycle attests that the final temperature determines the resultant microstructure and the associated residual stress. As a consequence, the residual stress can be analyzed to conclude on the impact of thermal stress contribution. Regarding the impact of sintering temperature, as expected, the lower the sintering temperature is, the lower the residual stress will be. While some relaxation of the stress can be expected for porous microstructures with respect to the denser ones, one could argue that the presence of local stress peaks in correspondence of microdefects may give an even lower toughness of the materials. Nonetheless, our measurements show that average microstrains in the film decrease upon curing. The temperature indeed tends to eliminate crystalline defects and especially decreases the density of twins.

This healing phenomenon can be investigated using a high-resolution XRD setup in combination with a Warren-Averbach procedure. In this treatment, the respective effects of crystallite size, twin fault density and microstrains on diffraction peaks are derived through Fourier analysis of the peaks. As given in Table 2, the initial twin fault density $\beta$ is significant within the nanoparticles. A high density of crystal defects is indeed generated during nanoparticle fabrication based on mechanical attrition [19]. Since the stacking fault energy of silver is very low $\left(18 \mathrm{~mJ} \cdot \mathrm{m}^{-2}\right)$, the external mechanical impact on nanoparticles can be accommodated through the formation of crystal twins. In parallel, in the initial state there are relatively large lattice distortions in the Ag nanoparticles compared to the cured state, which is probably the consequence of their decahedron shape [20]. This shape results from the interplay between surface tension and internal strain to reduce the free energy of the nanoparticles. It is known that a decahedron can be built from five tetrahedrons by accommodating some internal strain [20]. Microstrain is anisotropic and is higher in the $\{200\}$ direction.

Table 2: Average crystallite size $D$, twin fault density $\beta$, average microstrain (in $<111>$ and $<200>$ directions) and their standard errors as a function of curing temperature.

\begin{tabular}{c|cccc}
\hline $\begin{array}{c}\text { Curing temperature } \\
{\left[{ }^{\circ} \mathrm{C}\right]}\end{array}$ & $D[\mathrm{~nm}]$ & $\beta[\%]$ & $\left\langle\varepsilon_{L}^{2}\right\rangle_{111}^{1 / 2} \cdot 10^{4}$ & $\left\langle\varepsilon_{L}^{2}\right\rangle_{200}^{1 / 2} \cdot 10^{4}$ \\
\hline 20 & $13 \pm 1$ & $1.8 \pm 0.16$ & $30 \pm 2$ & $40 \pm 11$ \\
150 & $18 \pm 2$ & $1.6 \pm 0.10$ & $7 \pm 1$ & $21 \pm 8$ \\
300 & $96 \pm 10$ & $0.8 \pm 0.05$ & $4 \pm 4$ & $9 \pm 4$ \\
500 & $160 \pm 20$ & $0.3 \pm 0.02$ & $2 \pm 0.8$ & $3 \pm 0.5$ \\
\hline
\end{tabular}

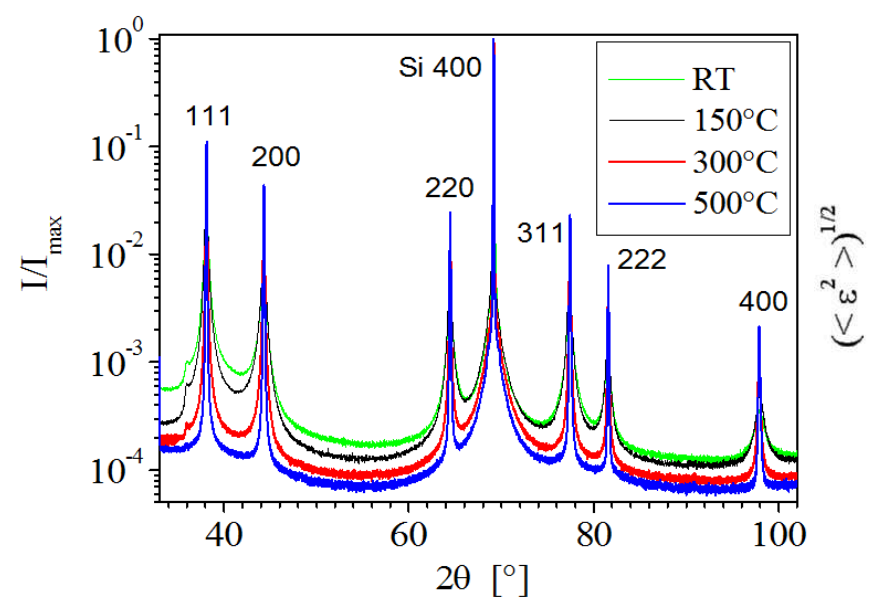

(a)

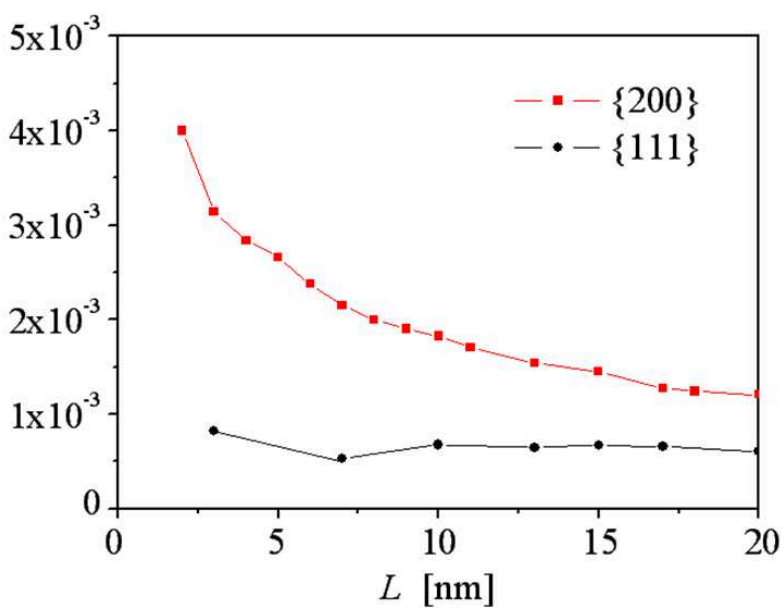

(b)

Figure 3: High-resolution diffraction peaks recorded on silver nanoparticle thin-films after curing at $150^{\circ} \mathrm{C}, 300^{\circ} \mathrm{C}$ and $500^{\circ} \mathrm{C}$ (a), and mean quadratic microstrain in the silver nanoparticles cured at $150^{\circ} \mathrm{C}$ as a function of the Fourier parameter $\mathrm{L}(\mathrm{b})$. 
Curing Ag nanoparticles causes crystallite growth and lattice strain relaxation together with a decrease in twin fault density. $\beta$ varies from an initial value of $1.8 \%$ at room temperature to $0.3 \%$ at $500{ }^{\circ} \mathrm{C}$. This corresponds respectively to 56 and 333 crystal planes in-between twin faults. The values and the evolution of $\beta$ are consistent with literature for multiply twinned particles. Previous experiments reported on gold nanoparticles (very close to Ag crystallographic structure) of $5 \mathrm{~nm}$ and $10 \mathrm{~nm}$ diameter led to values of $3.6 \%$ and $6.5 \%$, respectively [21,22]. Regarding crystallite size, this is quite stable up to $150^{\circ} \mathrm{C}$ and is the result of the solid-state sintering mechanism through interparticle neck growth. This neck growth can be formed through competitive processes via surface, lattice and grain boundary diffusion [23]. As previously reported, this sintering phenomenon is also associated with an improved electrical conduction as the curing temperature is increased [24]. Further annealing above $150^{\circ} \mathrm{C}$ leads to the growth of large grains and to crystallographic defect recovery. At $500^{\circ} \mathrm{C}$, the lattice strain becomes nearly isotropic.

\section{Conclusion}

X-ray diffraction has been successfully deployed for the multi-scale analysis of nanoparticle-based thin films. Despite the complexity of polycrystalline nanoparticles with an evolutive microstructure during a thermal process, X-ray peaks' broadening was correlated to crystallite growth, microstrain and internal stress. The different measurements are consistent with a significant decrease of microstrains during sintering, while no threading dislocation nor structural defect are created during cooling down as it would result in microstrains. Unlike conventional PVD thin films, inkjet-printed films thus exhibit an average residual stress below $100 \mathrm{MPa}$. An in-situ stress analysis further showed the characteristic temperatures from which a sintering scenario has been developed for nanoparticles. This multi-scale approach using the Warren-Averbach and the $\sin ^{2} \psi$ methods can suitably be used for understanding the effect of particle size and incident thermal energy sources (infrared, visible light, UV, microwave) on the sintering kinetics and mechanisms.

\section{References}

[1] R. Vayrette, C. Rivero, S. Blayac, K. Inal, Mater. Sci. Forum 681 (2011) 139.

[2] M. Gelfi, E. Bontempi, R. Roberti, L. Armelao, L.E. Depero, Thin Solid Films 450 (2004) 143.

[3] T. Wermelinger, S.A. Scott, M.G. Lagally, et al., AIP Conf. Proc. 1267 (2010) 776.

[4] R. Parashkov, E. Becker, T. Riedl, H.-H. Johannes, W. Kowalsky, Proc. IEEE 93 (2005) 1321.

[5] J.B. Szczech, C.M. Megaridis, et al., IEEE Trans. Electron. Packag. Manuf. 25 (2002) 26.

[6] V. Pekkanen, M. Mäntysalo, K. Kaija, et al., Microelectron. Eng. 87 (2010) 2382.

[7] J.-W. Kim, Y.-C. Lee, J.-M. Kim, W. Nah, et al., Microelectron. Eng. 87 (2010) 379.

[8] E. Roduner, Chem. Soc. Rev. 35 (2006) 583.

[9] J.T. Lue, in: H.S. Nalwa (Ed.), Encycl. Nanosci. Nanotechnol., ASP, 2007, pp. 1-46.

[10] W. Luo, W. Hu, S. Xiao, J. Phys. Chem. C 112 (2008) 2359.

[11] C. Kirchlechner, K.J. Martinschitz, R. Daniel, et al., Thin Solid Films 518 (2010) 2090.

[12] R. Resel, E. Tamas, B. Sonderegger, P. Hofbauer, J. Keckes, J. Appl. Crystallogr. 36 (2003) 80.

[13]B.E. Warren, B.L. Averbach, J. Appl. Phys. 21 (1950) 595.

[14] A. Yakoub, M. Saadaoui, R. Cauchois, J.-M. Li, et al., in: Mater. Res. Soc. Fall Meet., 2011.

[15] T.C. Hodge, et al., IEEE Trans. Components, Packag. Manuf. Technol. A 20 (1997) 241.

[16] J.R. Greer, Nat. Mater. 12 (2013) 689.

[17] E. Eiper, J. Keckes, K.J. Martinschitz, I. Zizak, M. Cabié, et al., Acta Mater. 55 (2007) 1941.

[18] S. Seel, J. Hoyt, E. Webb, J. Zimmerman, Phys. Rev. B 73 (2006) 245402.

[19]D. Oleszak, P.H. Shingu, J. Appl. Phys. 79 (1996) 2975.

[20] T.P. Martin, Phys. Rep. 273 (1996) 199.

[21]B. Ingham, T.H. Lim, C.J. Dotzler, A. Henning, et al., Chem. Mater. 23 (2011) 3312.

[22] W. Vogel, J. Bradley, O. Vollmer, I. Abraham, J. Phys. Chem. B 102 (1998) 10853.

[23] M.A. Asoro, D. Kovar, Y. Shao-Horn, L.F. Allard, et al., Nanotechnology 21 (2010) 025701.

[24] R. Cauchois, M. Saadaoui, A. Yakoub, K. Inal, B. Dubois, et al., J. Mater. Sci. (2012). 